\title{
Small synchronous machine protection during voltage sags caused by MV grid faults
}

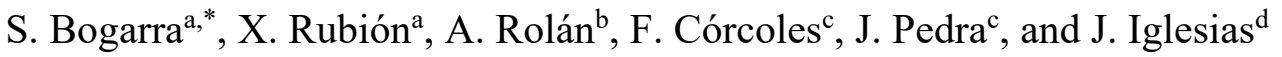 \\ a Department of Electrical Engineering, ETSEIAT-UPC, Colom 1, Terrassa-Barcelona 08222, Spain. \\ ${ }^{\mathrm{b}}$ Department of Industrial Engineering, Institut Químic de Sarrià - Ramon Llull University, Via Augusta \\ 390, Barcelona 08017, Spain. \\ ${ }^{\mathrm{c}}$ Department of Electrical Engineering, ETSEIB-UPC, Av. Diagonal 647, Barcelona 08028, Spain. \\ d PSGX Grid Systems, Asea Brown Boveri S.A., San Romualdo 13, Madrid, 28037, Spain. \\ *Corresponding author: Tel.: +34 93 7398037, fax: +34 937398236 \\ E-mail addresses: bogarra@ee.upc.edu, alejandro.rolan@iqs.url.edu,corcoles@ee.upc.edu, \\ pedra@ee.upc.edu, javier.iglesias@es.abb.com
}

\begin{abstract}
This paper analyses the impact of symmetrical and unsymmetrical voltage sags caused by distribution and transmission faults on the dynamic behaviour of small salient-pole synchronous machines connected to the MV grid, and proposes the modification of the protection settings to increase machine availability. As faults are considered, the voltage recovery is assumed to occur in a discrete way, in the fault current zeroes. The voltage sag effects include current and torque peaks, speed and load angle increase, and possible machine instability. Results from many cases are discussed, especially the positive-sequence voltage influence on machine stability. The study concludes that machine availability during unsymmetrical sags can be increased by modifying the undervoltage and overcurrent protection settings (the former should be based on the positive-sequence voltage instead of line-to-line voltages used in current settings).
\end{abstract}

Keywords: fault current, machine stability, overcurrent protection, synchronous machine, undervoltage protection, voltage sag

\section{Introduction}

The number of distributed generation (DG) units, such as wind turbines, photovoltaic generators and combined heat and power (CHP or cogeneration) plants, has greatly increased over the recent years. Small units are usually connected to the distribution 
system, i.e., to MV grids. Small CHP plants consist of a heat engine as a prime mover and a synchronous machine (SM) as a generator, whose rated power is usually in the range of 1-5 MW, which corresponds to the machine considered in this study.

Most grid codes do not contain fault ride-through requirements for DG units connected to the MV grid, and some codes even stipulate that these generators must disconnect during a disturbance. However, with a future larger amount of DG it will become necessary to maintain continuous generator operation, so the grid operators (in both transmission and distribution systems) should update their grid codes in order to face with the grid's integration of distributed systems [1], as stablished by the Regulation (EU) 2016/631 [2]. Additionally, [3] recommends the need for adopting the minimum tolerance thresholds in the IEEE Std. 1547 [4]. To achieve that goal, the determination of SM stability and dependence on the system parameters and variables is critical to avoid unnecessary grid disconnections.

This paper contains two studies: (1) the impact of voltage sags caused by distribution and transmission faults on SMs connected to the MV grid is exhaustively analysed, including the machine stability evaluation, and (2) the modification of the protection settings is proposed in order to increase machine availability. As there is a wide range of choices for both analyses, the study focuses on providing qualitative results. These analyses are based on the system represented in Fig. 1. The power plant is composed by a SM whose prime mover is a gas turbine, a power transformer, and the undervoltage and overcurrent protections (UV and OC, respectively). The considered excitation system is brushless with PM pilot exciter. The machine data is included in Table A.1.

The faulted power system in Fig. 1 is modelled as a controlled voltage source, which allows considering (by means of parametric analysis; sag type, duration and depth) all fault types produced at any distance from the machine, as well as sag propagation 
through all possible transformer connections. Sag effects on the SM include current and torque peaks, speed and load angle increase, and possible machine instability. These effects are obtained along this paper by assuming that the excitation voltage and the mechanical torque of the SM remain constant during the entire event. This assumption is justified because the influence of the automatic voltage regulator (AVR) and the prime mover control is negligible on the current and torque peaks and speed increase, while the influence on the load angle increase is always in the sense of predicting a more stable SM behaviour. A brief analysis on the excitation system and prime mover influence on the load angle is shown in Fig. $4 c$ of subsection 3.4.

Estimation of current and torque peaks due to sags has been done in the literature assuming that the voltage recovery after a fault is done instantaneously in all faulted phases [5]-[6]. Such fault clearance approach provides excessive overestimation of both current and torque peaks. A more realistic approach is considered in this paper: the voltage is recovered at the zero-crossing instants of the fault current, resulting in accurate estimation of these peaks. The analysis of a wide range of sag durations and depths, for symmetrical and unsymmetrical sags, provides the maximum duration of the fault before the machine loses its stability (critical clearing time). The study has let us obtain the critical clearing time (CCT) curves for all cases, which allows determination of the machine stability.

In [7]-[10] the settings in the protection equipment are studied under symmetrical sags. These studies have shown that changing the settings may reduce the number of undesired shutdowns. Ref. [8] reports that the UV margin of protection is excessive and proposes different UV settings for each specific type of DG unit based on transient stability studies. In [7] new UV settings of CHP plants connected to a MV grid structure are proposed based on voltage dip profiles. It should be noted that in previous 
references [1]-[3], [7]-[10] there is no distinction between the fault types in the trip requirements curve. On the contrary, this paper takes into account all sag types, which allows concluding that the minimum tolerance thresholds could be reduced for the sags due to unsymmetrical faults, which are the most frequent. Lastly, this paper proves that the machine availability is improved when UV trip is based on the positive-sequence voltage. The $\mathrm{OC}$ protection setting can also be modified to enhance the machine connection time.

Subsection 3.4 shows that a detailed model for the AVR or the prime mover (instead of the approaches in Fig. 1) results in an improved CCT and a large margin of protection, which reinforces the idea of protection settings modification to increase SM availability. This subsection also shows the influence of d-axis reactance and mechanical inertia on the CCT curve.

The procedure proposed in this paper can be followed to determine the margin of protection of any considered power plant. It is expected that protection settings may be modified to increase machine availability during sags.

\section{Sag classification and characterization}

\subsection{Sag classification}

As shown in Fig. 1, the SM terminals are connected through a transformer to the point of common coupling (PCC). The faulted power system (as seen by the PCC) is emulated by a controlled voltage source. As shown later, this voltage source includes the different fault types and the different transformers (with all the possible connections) between the PCC and the point where the fault is produced. Sag propagation depends on the transformer connections as shown in Table 1 for two cascade transformers. 
Only Yd or Dy transformers are taken into account because they are the ones that lead to new sag types [11]. For example, a two-phase fault causes a type $\mathrm{C}$ sag at the faulted voltage level; this sag type does not change when passing through YNyn, Yy or Dd transformers, but develops into a type $\mathrm{D}$ sag when passing through $\mathrm{Yd}$ or Dy transformers. This type D sag is not modified when passing through YNyn, Yy or Dd transformers, while it changes again to a type $\mathrm{C}$ sag when transformers are $\mathrm{Yd}$ or Dy.

The due-to-fault sag classification proposed in [12] divides sags into fourteen types (from $A_{1}$ to $G_{2}$ ), which are the result of considering the influence of the different transformer connections on sag propagation throughout the power system (Table 1, [11]-[12]). According to this classification, the faults that cause type A sags can be cleared in five different ways $\left(\mathrm{A}_{1}, \mathrm{~A}_{2}, \mathrm{~A}_{3}, \mathrm{~A}_{4}\right.$ and $\left.\mathrm{A}_{5}\right)$ involving two or three steps. The faults that cause type E, F and G sags can be cleared in two different ways ( $E_{1}$ and $E_{2}$; $F_{1}$ and $F_{2} ; G_{1}$ and $G_{2}$ ) involving two steps. Finally, the faults that cause type $B, C$ and $D$ sags are cleared in only one way involving a single step. As fault clearing occurs in the fault current zeros, the voltage recovers at discrete instants, which depend on the fault current angle, $\psi$. The fault current angle is defined in [12] as: "Let $\cos (\psi)$ be the power factor of the fault current then the current zero crossing takes places at an angle $\psi$ for the pre-fault voltage". In turn, this angle depends on the type of network: the typical range is between $75^{\circ}$ and $85^{\circ}$ for faults in the transmission system, and between $45^{\circ}$ and $60^{\circ}$ for faults in the distribution system. The values chosen for the examples in this paper are $\psi=80^{\circ}$ and $\psi=52^{\circ}$ for faults in the transmission and distribution systems, respectively.

To sum up, the consideration of all sag types in the controlled voltage source of Fig. 1 is equivalent to consider all fault types and all possible transformer connections (as seen from the PCC). 
Two aspects must be considered about the power plant transformer influence on the machine behaviour: (1) the transformer impedance, and (2) the transformer connections. As the transformer impedance is smaller than the SM d-axis reactance, its effect can be neglected. Regarding the different transformer connections, for a given sag type it is indifferent to consider or to neglect the transformer connections, as identical abc currents (in pu) at the PCC and identical SM dynamic behaviour are obtained in both cases. For example, a type C sag at the PCC through a Dy9 transformer is seen by the $\mathrm{SM}$ as a type D sag, and the evolution of all dynamic variables (in pu) is identical to the case when the same type C sag is directly applied to the SM terminals, as shown in [13]. Regarding abc currents (in pu) at the PCC, it can be proved that they are identical with or without transformer when the same type $\mathrm{C}$ sag is applied at the PCC. In short, the set SM-transformer (as seen from the PCC) is equivalent to consider just the SM when pu variables are used.

\subsection{Sag characterization}

The parameters that univocally characterize sags are type, depth $h$, duration $\Delta t$ and angle $\psi$. Other useful, but not univocal, parameters are phase angle of phase A voltage $\alpha_{\mathrm{a}}$ and initial and final points-on-wave $\gamma_{\mathrm{i}}$ and $\gamma_{\mathrm{f}}$, all of which are related to the pre-event voltage:

$$
v_{\mathrm{a}}(t)=\sqrt{2} V_{\mathrm{a}} \cos \left(\omega t+\alpha_{\mathrm{a}}\right) \rightarrow \begin{aligned}
& \gamma_{\mathrm{i}} \\
& =\omega t_{\mathrm{i}}+\alpha_{\mathrm{a}} \\
& \gamma_{\mathrm{f}}=\omega t_{\mathrm{f}}+\alpha_{\mathrm{a}} \\
& \gamma_{\mathrm{i}}=\gamma_{\mathrm{f}}-\omega \Delta t
\end{aligned}
$$

where $v_{\mathrm{a}}$ is the instantaneous phase A voltage, $V_{\mathrm{a}}$ is the rms phase A voltage, $\omega$ is the network angular frequency, $t_{\mathrm{i}}$ is the instant of fault initiation and $t_{\mathrm{f}}$ is the instant of fault clearing (i.e., the instant when the first phase is recovered; thus, $\gamma_{\mathrm{f}}$ is also referred to as 
the fault clearance angle). In all simulations of this paper, it is assumed that $\alpha_{a}=0^{\circ}$. Table 2 shows the angle $\gamma_{f}$ for all sag types (i.e., taking into account all transformer connections), and for the two values considered for angle $\psi$. It must be noted that angle $\gamma_{\mathrm{f}}$ only depends on the sag type and on angle $\psi$ :

$$
\gamma_{\mathrm{f}}=\text { function }(\text { sag type }, \psi)
$$

In this paper, the sag shape is regarded as piecewise rectangular (discrete sag in Fig. 1), resulting in a voltage recovery process which can involve more than one step depending on the number of faulted phases. Discrete sags include the fault clearing process, which leads to the realistic $\gamma_{\mathrm{f}}$ values of Table 2. A less realistic model (usually assumed in the literature [5]) is the abrupt sag model in Fig. 1 (the voltage recovery process is done in one step), which assumes that the fault is cleared abruptly in all affected phases. The abrupt model is disregarded in this paper because it results in excessive overestimation for both current and torque peaks.

\section{Impact of voltage sags}

\subsection{Synchronous machine dynamic behaviour}

Sag effects on SM behaviour have been fundamentally studied by simulation in the literature [5]-[10], as dynamic behaviour testing of SM around several MVA is not a very common task. Ref. [14] is an exception to this, as the ride-through of a MV SM working as a motor is analysed through simulated and experimental results.

SMs can ride-through voltage sags, helping the main grid to recover the pre-event voltage level. For this reason, it is important to know the sag influence on SM behaviour. The machine behaviour depends on many factors such as $h, \Delta t, \gamma_{i}, \psi$, sag type, SM parameters, operating point and mechanical inertia. Observed effects (irrespective of the sag type) include: 
- stator current increase, which can trigger OC protection,

- electromagnetic torque peaks, which exceed steady-state stress levels and can cause a fatigue failure, and

- speed and load angle increase, which can cause SM instability.

Fig. 2 exemplifies SM behaviour due to a type $A_{1}$ sag (symmetrical) and a type $E_{1}$ sag (unsymmetrical), both with $h=0.1$ and $\psi=52^{\circ}$, and $\Delta t=19.64 T(327.3 \mathrm{~ms}$ ) and $\Delta t=19.81 T(330.2 \mathrm{~ms})$, respectively. The analysis of Fig. 2 and of many other sags (not included here for brevity) allows the following conclusions to be drawn:

- The maximum current and torque peaks are obtained at voltage recovery only in a small number of cases: symmetrical sags with depth and duration driving the SM to near instability. As the instant of fault clearance depends on $\psi,(2)$, these maximum peaks depend on $\psi$ and fault clearing process. This is the case of Fig. $2 a$.

- The maximum current and torque peaks are obtained at sag initiation in the remaining sags: all unsymmetrical sags and virtually all symmetrical sags. As the instant of fault initiation is arbitrary, these maximum peaks depend on $\gamma_{\mathrm{i}}$, but are not influenced by $\psi$ or the fault clearing process. This is the case of Fig. $2 b$.

- The speed and load angle increase, and SM stability are not influenced by $\gamma_{i}, \psi$ or the fault clearing process. Thus, it is not necessary to differentiate between the several subtypes (i.e., subtypes $A_{1} \ldots A_{5}$ are grouped as type $A$; subtypes $E_{1}, E_{2}$ as type $E_{\ldots .}$ ) to evaluate these effects.

\subsection{Fault clearance and fault current angle influence on current and torque peaks}

When sag is modelled discrete and the values for $\gamma_{\mathrm{f}}$ in Table 2 are used, the most severe current and torque peaks are obtained at sag initiation for all unsymmetrical sags and for virtually all symmetrical sags, as said in the previous subsection. On the contrary, when sag is modelled abrupt and/or unrealistic values for $\gamma_{\mathrm{f}}$ are considered, as 
in [5], the maximum current and torque peaks are largely overestimated as they are obtained at voltage recovery.

For the remaining sags (symmetrical sags with depth and duration driving the SM to near instability), $\psi=52^{\circ}$ (for faults in the distribution system) leads to more severe current and torque peaks at voltage recovery than $\psi=80^{\circ}$. Where appropriate, $\psi=52^{\circ}$ will be used in the rest of the paper.

To sum up, if discrete sags and the realistic values for $\gamma_{\mathrm{f}}$ in Table 2 are considered, the fault clearing process and angle $\psi$ do not influence the most severe current and torque peaks in most sag events.

\subsection{Initial point-on-wave and depth influence on current and torque peaks at sag initiation}

Angle $\gamma_{\mathrm{i}}$ (related to the instant of fault initiation) and sag depth have a strong influence on the current and torque peaks at sag initiation. As the fault clearing process does not influence these peaks, it is not necessary to differentiate between subtypes.

Fig. $3 b$ shows the periodical influence of $\gamma_{i}$ on the current (solid line) and torque (dashed line) peaks at sag initiation for all sag types when depth is $h=0.1$. For the symmetrical sags, $\gamma_{i}$ has a negligible influence on the current peaks (a $\pi / 3$ periodicity is observed), and no influence on the torque peaks. On the contrary, it has a large influence on the current and torque peaks of unsymmetrical sags (a $\pi$ periodicity is observed). The numerical results of Fig. $3 b$ are summarized in Table 3. For example, the maximum current peak for a type A sag is $i_{\mathrm{s} \max }=9.18 \mathrm{pu}$ and appears at $\gamma_{\mathrm{i}}=33.5^{\circ}$, and the minimum current peak is $i_{\mathrm{s} \min }=8.74 \mathrm{pu}$ and appears at $\gamma_{\mathrm{i}}=3.5^{\circ}$. However, the maximum and minimum torque peaks for this sag type are $T_{\mathrm{e} \max }=T_{\mathrm{e} \min }=6.11 \mathrm{pu}$, regardless of $\gamma_{i}$, i.e., $\gamma_{i}$ has no influence on the torque peaks of symmetrical sags. 
The depth influence on the current and torque peaks for all sag types is shown in Fig. $3 a$ and Fig. 3c, respectively. The almost linear dependence in all cases is apparent. The following surprising observations can also be made:

- for a given $h$, and according to the value of $\gamma_{i}$, a two-phase fault (sag types C and D) can produce stronger torque peaks than a three-phase fault (sag type A) or a twophase-to-ground fault (sag types E, F and G),

- for a given sag type, and according to the value of $h$ and $\gamma_{i}$, a shallower sag (higher $h$ ) can produce stronger current and torque peaks than a deeper sag (lower $h$ ).

\subsection{Synchronous machine stability. Influence of AVR, prime mover control and main parameters}

The authors have verified that the influence of the automatic voltage regulator and the prime mover control is negligible on the current and torque peaks and speed increase. The influence on the load angle increase is always in the sense of predicting a more stable SM behaviour. For this reason, the excitation voltage and the mechanical torque of the SM have been assumed constant in all the study but other assumptions have been made in Fig. 4c to show that the SM stability is improved. Consequently, as the aim of this study is to show that the SM availability is improved when UV trip is based on the positive-sequence voltage, the assumption corresponds to a more unfavourable situation.

A measure of SM stability is the CCT [7]-[10]. For a sag of a certain depth, the CCT is the maximum duration of the sag which can be ridden-through by the machine, i.e., the machine is capable of returning to pre-event stable operation. Fig. $4 a$ and Fig. $4 b$ summarize the CCT curves for all sag types assuming that the excitation voltage and the mechanical torque remain constant during the entire event. A sag with depth and 
duration within the area bounded by its CCT curve leads to unstable operation of the SM. Symmetrical sags have shorter CCTs than unsymmetrical sags with the same depth.

Sags are grouped according to their severity: (1) symmetrical sags; (2) unsymmetrical type E, F and G sags; (3) unsymmetrical type C and D sags, and (4) unsymmetrical type B sag, under which the SM is capable of remaining in stable operation, irrespective of depth and duration

As SM stability depends on the positive-sequence voltage, $V_{1}$ (the negative- and the zero-sequence voltages have almost no influence), another sag severity classification is according to voltage $V_{1}$. The sequence voltages of all sag types are listed in Table 4 .

The CCT curves for all sag types using $V_{1}$ for ordinates are superimposed in Fig. $4 b$. Since SM stability depends on $V_{1}$, the CCT curves of the unsymmetrical sags are identical to those of the symmetrical ones, except for some negligible differences. Two examples marked with a triangle and a circle in Fig. $4 a$ and Fig. $4 b$ illustrate this feature:

- the point marked with a triangle: the CCT of a type A sag with depth $h=0.51 \mathrm{pu}\left(V_{1}\right.$ $=0.51 \mathrm{pu})$ is $139.5 T(2.325 \mathrm{~s})$. The CCTs of the type E, F and G sags with depth $h=$ $0.27 \mathrm{pu}\left(V_{1}=0.51 \mathrm{pu}\right)$ are also $139.5 T$. Finally, the CCTs of the type C and D sags with depth $h=0 \mathrm{pu}\left(V_{1}=0.51 \mathrm{pu}\right)$ are again $139.5 T$.

- the point marked with a circle: the CCT of a type A sag with depth $h=0.34 \mathrm{pu}$ $\left(V_{1}=0.34 \mathrm{pu}\right)$ is $43.1 T(718.3 \mathrm{~ms})$. The CCTs of the type E, F and G sags with depth $h=0 \mathrm{pu}\left(V_{1}=0.34 \mathrm{pu}\right)$ are also $43.1 T$.

Fig. $4 b$ also shows that the SM operation remains stable for any sag with $V_{1}$ above $0.55 \mathrm{pu}$. This is because the type B sag does not put SM stability at risk (the minimum voltage for a type B sag is $V_{1}=0.67 \mathrm{pu}$, see Table 4). 
Next, the influence of the excitation system and mechanical torque approaches on the CCT curve are briefly analysed. The exciter and AVR are represented by a Type AC8B model and Type 2 var controller [15] (the chosen parameters are based on the sample data provided by the standard), while the prime mover is a gas turbine. Fig. $4 c$ shows the CCT curves for next cases: (1) a constant value for both excitation voltage and mechanical torque, (2) AVR and constant mechanical torque, and (3) AVR and prime mover control. The figure shows that SM stability is improved when controlling either the excitation or the mechanical torque, or when controlling both [16].

Lastly, Fig. $4 d$ and Fig. $4 e$ show the influence of the d-axis reactance and the mechanical inertia values on the CCT curve. It is shown that a large d-axis reactance (or the consideration of the transformer reactance) and a large inertia increase the SM instability.

\section{Sensitivity curves}

The sensitivity of electrical components to voltage sags is usually represented by sensitivity curves, which are in terms of the depth and duration of the voltage sag [11]. Although the $h$ and $\Delta t$ influence on SM behaviour can be illustrated with the threedimensional (3D) surfaces of Fig. 5a, the most usual representation is as level curves in magnitude-duration plots, as can be seen in Fig. $5 b$.

These level curves are horizontal cut-outs of the 3D surface at different levels and represent SM sensitivity to sags. These curves show the way a voltage sag with a specific depth and duration affects the SM behaviour through the analysis of several variables, and they facilitate the determination of the variable reaches a value higher than the threshold or leads to instability. Only the points at which the SM remains in stable operation are represented in Fig. $5 b$ whereas the unstable operation points fall 
within the shaded area.

The magnitudes chosen to characterize SM sensitivity are:

- maximum current peak, is max;

- maximum electromagnetic torque peak, $T_{\mathrm{e} \text { max; }}$

- time overcurrent exceeds $2 \mathrm{pu}$, toc $2 \mathrm{pu}$ : time interval in which the rms line current exceeds twice the rated current. This magnitude informs about the definite time OC protection trip with the settings of Fig. $6 a$. Other settings can be chosen, such as those in Fig. $6 c$ (explained in next section), and

- maximum speed, $\omega_{\mathrm{m} \text { max. }}$

Simulations of all sag types were carried out, but only sag types $A_{1}, E_{1}$ and $C$ are shown in Fig. 5b. The most unfavourable values of $\gamma_{i}$ (see Table 3) are used for the simulations. The sag subtypes are not considered for calculating the toc and $\omega_{\mathrm{m} m a x}$ curves. The analysis of Fig. 5 reveals that

- The maximum torque peak, $T_{\mathrm{e} \text { max }}$, for a type $\mathrm{C}$ sag is more severe than for types $\mathrm{E}_{1}$ and $\mathrm{A}_{1}$ (as previously predicted in Fig. 3).

- The toc curves are useful for the OC setting. For example, if the setting is $2 \mathrm{pu}-0.3 \mathrm{~s}$, a trip will occur for type $\mathrm{C}$ sags deeper than $0.55 \mathrm{pu}$, type $\mathrm{E}_{1}$ sags deeper than $0.50 \mathrm{pu}$ and type $\mathrm{A}_{1}$ sags deeper than $0.45 \mathrm{pu}$.

- A peak appears in the $\omega_{\mathrm{m} \text { max }}$ curves for durations shorter than $15 T$ (250 ms) because the instant of voltage recovery coincides with the first speed oscillation.

\section{Machine availability increase by UV and OC setting modification}

Synchronous generators make use of different protective functions provided by individual component relays or integrated into MGPS (Multifunction Generator Protection Systems) packages [20], but in this paper, only the effect of the MV grid faults on the SM stability is analysed. So, the UV and OC protections are considered 
[7]. For the SM the CCT are obtained and the effect of protection setting is studied.

\subsection{Current margin of protection with $O C_{1}$ and $U V_{1}$ settings}

In this study it has been assumed that the local utility requires the OC and UV protections (ANSI codes 51 and 27, respectively) installation at the PCC in Fig. 1, as is the case of the Spanish Utilities. The UV protection settings are imposed by the utility, while the OC protection settings are typical values providing a wide safety margin with respect to the machine limits. New settings are proposed with the aim of maintain continuous machine operation (if possible) in case of an external fault.

The following usual UV and OC settings are considered in this subsection to illustrate the present margin of protection of the SM:

- UV 1 setting of Fig. $6 b$ (IEEE Std. 1547 [4]), which is based on the line-to-line voltages, i.e., a trip occurs if any of the three line-to-line voltages falls below the limit value. Fig. $6 b$ also includes two other UV settings which have not been considered in this paper [17]-[18].

- $\mathrm{OC}_{1}$ setting of Fig. $6 c$, which is composed of the superposition of the definite time curves $1.2 \mathrm{pu}-1 \mathrm{~s}$ and $2 \mathrm{pu}-0.3 \mathrm{~s}$.

Fig. $7 a$ contains the following curves for sag types A, E and C: (1) the $\mathrm{UV}_{1}$ trip curve of Fig. $6 b$, (2) the $\mathrm{OC}_{1}$ trip curve calculated for the settings of Fig. $6 c$, and (3) the CCT curve of Fig. $4 b$ (with a logarithmic scale for abscissae). It is worth noting that the $\mathrm{OC}_{1}$ trip curve in Fig. $7 a$ is similar to the toc 2 pu -0.3 s curve in Fig. 5 because of the similarity between the corresponding setting curves in Fig. $6 a$ and $c$.

Fig. $7 a$ shows that the protection settings cause SM disconnection before instability. While the settings seem adequate for the symmetrical sags (type $A_{1}$ ), there is overprotection of the unsymmetrical ones (especially type C). For example, $\mathrm{UV}_{1}$ setting causes disconnection in 10 cycles $(167 \mathrm{~ms})$ for type $\mathrm{C}$ sags deeper than $0.5 \mathrm{pu}$ although 
the machine could remain connected from the stability point of view. This margin of protection can be regarded as excessive considering that the probability of exposure to unsymmetrical sags is around $80 \%$ [19].

\subsection{Proposed $U V_{2}$ and $\mathrm{OC}_{2}$ settings to increase the connection time}

The simulation shows that DG-units are disconnected with a wide margin before the SM instability is achieved, especially for unsymmetrical sags. So, in a scenario with high DG penetration in which it is necessary to feed local loads a modification of the settings will be required. As a result, to increase the SM availability and reduce the fault clearing time, new settings are considered.

This subsection analyses the following proposed settings to increase the connection time during unsymmetrical sag events:

- $\mathrm{UV}_{2}$ setting, which is identical to the $\mathrm{UV}_{1}$ setting but using the positive-sequence voltage instead of the minimum line-to-line voltage. That is, the settings in Fig. $6 b$ are maintained but the ordinate axis legend is changed to 'Minimum positive-sequence voltage (pu)'. Note that positive-sequence UV is commonly implemented in modern microprocessor based relays (ANSI code 27D).

$-\mathrm{OC}_{2}$ setting of Fig. $6 c$, which is comprised by the definite time curves $1.2 \mathrm{pu}-1.5 \mathrm{~s}$ and $2.5 \mathrm{pu}-0.3 \mathrm{~s}$. These limits are far enough from the thermal limits provided by SM manufacturers or from the standards [20].

Fig. $7 b$ plots the $\mathrm{UV}_{2}$ and $\mathrm{OC}_{2}$ trip curves, and the CCT curves for sag types $\mathrm{A}, \mathrm{E}$ and

C. The new $\mathrm{OC}_{2}$ curve is obtained from new toc sensitivity curves. The figure shows that the proposed settings increase SM availability by ensuring disconnection before instability and considering the manufacturer thermal limits.

Taking into account that most of grid faults are unsymmetrical and most of which are 
short-duration faults, the UV protection is the one that disconnects the SM from the grid. Then, the SM availability is improved when UV trip is based on the positivesequence voltage.

\section{Conclusions}

By considering the expected increase in the number of distributed generation units connected to $\mathrm{MV}$, the behaviour of a small SM during voltage sags caused by faults is studied in this paper. In this scenario, it will become necessary to maintain continuous generator operation as long as possible. Thus, the grid operators should update the national grid codes to address the integration of distributed generation systems. This goal requires the SM stability determination, and its dependence on the system parameters and variables is critical to avoid unnecessary disconnections from the grid. In the present study, a parametric analysis allows considering all fault types produced at any distance from the machine, as well as sag propagation through all possible transformer connections.

Despite voltage sag effects on the machine have been widely studied in the literature, this paper considers a more realistic approach, which assumes that voltage recovers at the zero-crossing instants of the fault current (discrete sags). When the SM is far from instability, the most severe current and torque peaks are obtained at sag initiation for specific initial points-on-wave. These peaks have periodic behaviour with the initial point-on-wave. Interestingly, the most unfavourable torque peaks are not produced for symmetrical sags (type A), but for the unsymmetrical ones caused by single-phase and phase-to-phase faults (types C and D). Also, for a given sag type, shallower sags can lead to stronger current and torque peaks than deeper sags for specific initial points-onwave.

CCT curves are calculated for symmetrical and unsymmetrical sag types. As SM 
stability depends on the positive-sequence voltage, all CCT curves are superimposed onto a single curve when such sequence voltage is used for ordinates.

Current UV and OC protection settings are usually based on symmetrical sags. This paper has proved that such protection settings produce unnecessary machine disconnections for many unsymmetrical sags (especially for types $\mathrm{C}$ and $\mathrm{D}$ ), which are the most frequent. Thus, these settings are inadequate when the goal is to maintain continuous generator operation as long as possible. SM availability during these sags can be increased by modifying both settings: UV trip must be based on the positivesequence voltage instead of line-to-line voltages and OC trip must be based on the proposed $\mathrm{OC}_{2}$ curve. Additional enhancements on the connection time are possible if less conservative OC settings are used.

\section{Acknowledgements}

The authors acknowledge the financial support of the Ministry of Science and Innovation of Spain through the project DPI2011-28021.

\section{References}

[1] T. Preda, K. Uhlen, D.E. Nordgard, An overview of the present grid codes for integration of distributed generation, CIRED Workshop, Lisbon, Portugal, May 2012, 1-4.

[2] Establishing a network code on requirements for grid connection of generators, Commission Regulation (EU) 2016/631, 2016.

[3] NERC: Performance of distributed energy resources during and after system disturbance, Technical Report, December 2013. 
[4] IEEE Standard for interconnecting distributed resources with electric power systems, IEEE Std. 1547, 2008.

[5] F. Carlsson, C. Sadarangani, Behavior of synchronous machines subjected to voltage sags of type A, B and E, EPE Journal 15 (4) (2005) 1-8.

[6] D. Aguilar, A. Luna, A. Rolán, G. Vázquez, G. Acevedo, Modeling and simulation of synchronous machine and its behaviour against voltage sags, IEEE International Symposium on Industrial Electronics, ISIE 2009, Seoul, Korea, July 2009, 729-733.

[7] E.J. Coster, J.M.A. Myrzik, W.L. Kling, Grid interaction of MV-connected CHPplants during disturbances, IEEE Power \& Energy Society General Meeting, PES '09, Calgary, Canada, July 2009, 1-8.

[8] I. Xyngi, M. Popov, Coordination between distributed generation stability and undervoltage protection requirements at DG interconnection point, International Conference on Power System Transients, IPST 2009, Kyoto, Japan, June 2009, 16.

[9] E.J. Coster, J.M.A. Myrzik, W.L. Kling, Transient stability of distributed generation in MV-ring networks, IEEE/PES Transmission and Distribution Conference and Exposition, IEEE PES T\&D 2008, Chicago, USA, April 2008, 17.

[10] J.C. Das, Effects of momentary voltage dips on the operation of induction and synchronous motors, IEEE Trans. Ind. Applications, 26 (4) (1990) 711-718.

[11] M.H.J. Bollen, Understanding power quality problems: voltage sags and interruptions, IEEE Press, New York, 2000.

[12] M.H.J. Bollen, Voltage recovery after unbalanced and balanced voltage dips in three-phase systems, IEEE Trans. Power Delivery, 18 (4) (2003) 1376-1381. 
[13] A. Rolán, F. Córcoles, J. Pedra, Ll. Monjo, S. Bogarra, Testing of three-phase equipment under voltage sags, IET Electr. Power Appl., 9 (4) (2015) 287-296.

[14] F. Endrejat, P. Pillay, Ride-through of medium voltage synchronous machine centrifugal compressor drives, IEEE Trans. Ind. Applications, 47 (4) (2011) 1567-1577.

[15] IEEE Recommended practice for excitation system models for power system stability studies, IEEE Std. 421.5, 2016.

[16] E.R. Collins, J. Jiang, Voltage sags and the response of a synchronous distributed generator: a case study, IEEE Transactions on Power Delivery, 23 (1) (2008) 442448.

[17] Technical conditions for electrical production installations connected to the Iberdrola distribution network, (in Spanish), Iberdrola, MT 3.53.01, 2012.

[18] Requirements for micro-generating plants to be connected in parallel with public low-voltage distribution networks, Cenelec, EN 50438, 2013.

[19] M.H.J. Bollen, et al., Voltage dip immunity of equipment and installations, CIGRE, Paris, France, CIGRE/CIRED/UIE Working Group C4.110, Tech. Broch. TB-412, Apr. 2010. Available online (Jul. 2016): http://www.uie.org/sites/default/files/generated/files/pages/CIGRE\%20TB412\%2 0voltage $\% 20 \mathrm{dip} \% 20 \mathrm{immunity} \% 20 \mathrm{of} \% 20$ equipment $\% 20$ and $\% 20$ installations.pdf.

[20] IEEE Guide for ac generator protection, IEEE Standard C37.102, 2006.

[21] EMTDC User Guide. Manitoba-HVDC Research Centre. Manitoba, Canada. Available (Jul. 2016): https://hvdc.ca/uploads/ck/files/EMTDC\%20Users\%20Guide\%20V4_6_0.pdf. 
[22] E. Levi, V.A. Levi, Impact of dynamic cross-saturation on accuracy of saturated synchronous machine models, IEEE Transactions on Energy Conversion, 15 (2) (2000) 224-230.

[23] E. Levi, Saturation modelling in $\mathrm{d}-\mathrm{q}$ axis models of salient pole synchronous machines, IEEE Transactions on Energy Conversion, 14 (1) (1999) 44-50.

[24] K.W. Louie, A study on magnetic saturation effects in a synchronous generator during unbalanced faults, International Conference on Power System Technology, PowerCon 2004, Singapore, November 2004, (2), 1496-1501.

[25] F. Carlsson, Saturation in synchronous machines due to voltage sags, IEEE International Electric Machines and Drives Conference, IEMDC'03, Madison (Wisconsin), USA, June 2003, (3), 1571-1575.

\section{Appendix: SM model and parameters}

The system of Fig. 1 is simulated in PSCAD/EMTDC. The used salient-pole SM is a built-in linear (non-saturated) model contained in the simulation package, whose mathematical description can be found in the chapter 7 of [21]. Support subroutines are included in the machine model library in PSCAD for calculating the equivalent circuit parameters of a synchronous machine from commonly supplied data.

The machine parameters of the SM studied are given in Table A.1. The SM operates as a generator at rated conditions before the sag, and for these operation conditions the mechanical torque is $0.9185 \mathrm{pu}$ and the excitation voltage is $2.4611 \mathrm{pu}$.

The built-in PSCAD/EMTDC model for the synchronous machine provides an average electromagnetic torque, so the instantaneous torque is calculated in this paper as: 


$$
T_{\mathrm{e}}=p\left\{M_{\mathrm{q}} i_{\mathrm{sd}} i_{1 \mathrm{q}}-\left(M_{\mathrm{d}} i_{\mathrm{f}}+M_{\mathrm{d}} i_{1 \mathrm{~d}}\right) i_{\mathrm{sq}}-\left(L_{\mathrm{d}}-L_{\mathrm{q}}\right) i_{\mathrm{sd}} i_{\mathrm{sq}}\right\}
$$

Fig. A.1 illustrates the large differences in the evaluation of the instantaneous and average torques for the sag in Fig. 2.

The calculations in this paper have been made by using the linear model of the SM, as no significant differences have been obtained when d-axis saturation model from PSCAD has been used. The inclusion of the saturation in the SM model has been widely studied in [22]-[23]. These studies show that the fact of considering the saturation has no significant effect on the peak values of the analysed magnitudes (lower than $10 \%$ ), although it can affect the dynamics of the transient behaviour. Reference [24] compares two saturation models for a large SM exposed to a one-phase-to-ground short circuit: daxis and both d- and q-axes saturation models. As the current peaks obtained in both cases are almost equal, the authors conclude that, in case that saturation must be taken into account, it is enough to consider d-axis saturation. References [5], [25] also consider saturation effect influence on the prediction of the machine behaviour exposed to sags. Despite using abrupt sag modelling (which overestimate current and torque peaks at voltage recovery), the authors state that the current and torque peaks after the sag are only a bit higher when saturation is included (compared with a linear model). 


\section{Figure captions}

Fig. 1. Diagram of the studied system.

Fig. 2. Machine behaviour due to sags of characteristics. (a) Type $A_{1}, h=0.10, \Delta t=19.64 T$ (327.3 ms), $\psi=52^{\circ} ;$ (b) type $\mathrm{E}_{1}, h=0.10, \Delta t=19.81 T(330.2 \mathrm{~ms}), \psi=52^{\circ}$

Fig. 3. Initial point-on-wave and depth influence on the current (solid blue line) and torque (dashed red line) peaks at sag initiation for all sag types. The initial point-on-wave influence is obtained for a depth $h=0.10$

Fig. 4. CCT curves for all sag types. Unless otherwise stated, excitation voltage and mechanical torque remain constant. (a) Using the sag depth, $h$, for ordinates; (b) using the positive-sequence voltage, $V_{1}$, for ordinates; (c) using $V_{1}$, for ordinates, and for three different control scenarios; (d) using $V_{1}$, for ordinates, and for different values of $d$-axis reactance; (e) using $V_{1}$, for ordinates, and for different values of inertia.

Fig. 5. Duration and depth influence. (a) Three-dimensional surfaces corresponding to current and torque peaks for sag type $A_{1}$, with $\psi=52^{\circ}$; (b) sensitivity curves for type $A_{1}$ symmetrical sags and for types $E_{1}$ and $\mathrm{C}$ unsymmetrical sags, all with $\psi=52^{\circ}$.

Fig. 6. Protection settings. (a) Settings for the definite time OC protection of Fig. 5; (b) undervoltage protection: current $\mathrm{UV}_{1}$ setting (IEEE Std. 1547) compared to Iberdrola and Cenelec settings; (c) overcurrent protection: current $\mathrm{OC}_{1}$ and proposed $\mathrm{OC}_{2}$ settings. 
Fig. 7. Trip curves considering sag types A, E and C. (a) Trip curves for current $U_{1}$ and $\mathrm{OC}_{1}$ settings compared to the CCT curves; (b) trip curves for the proposed $\mathrm{UV}_{2}$ and $\mathrm{OC}_{2}$ settings compared to the CCT curves.

Fig. A.1. Detail of the instantaneous torque given by (A.1) and average torque calculated by PSCAD/EMTDC for the type $\mathrm{A}_{1}$ sag in Fig. 2a.

\section{Table 2}

Final point-on-wave or fault clearance angle $\left(\gamma_{\mathrm{f}}\right)$.

\begin{tabular}{crrrrrr}
\hline Type & $\psi=52^{\circ}$ & $\psi=80^{\circ}$ & & Type & $\psi=52^{\circ}$ & $\psi=80^{\circ}$ \\
\cline { 2 - 5 } $\mathrm{A}_{1}$ & $-38^{\circ}$ & $-10^{\circ}$ & & $\mathrm{D}_{\mathrm{a}}$ & $-38^{\circ}$ & $-10^{\circ}$ \\
$\mathrm{A}_{2}$ & $52^{\circ}$ & $80^{\circ}$ & & $\mathrm{E}_{1 \mathrm{a}}$ & $82^{\circ}$ & $110^{\circ}$ \\
$\mathrm{A}_{3}$ & $-38^{\circ}$ & $-10^{\circ}$ & & $\mathrm{E}_{2 \mathrm{a}}$ & $-158^{\circ}$ & $-130^{\circ}$ \\
$\mathrm{A}_{4}$ & $52^{\circ}$ & $80^{\circ}$ & & $\mathrm{F}_{1 \mathrm{a}}$ & $172^{\circ}$ & $-160^{\circ}$ \\
$\mathrm{A}_{5}$ & $-38^{\circ}$ & $-10^{\circ}$ & & $\mathrm{F}_{2 \mathrm{a}}$ & $112^{\circ}$ & $140^{\circ}$ \\
$\mathrm{B}_{\mathrm{a}}$ & $-38^{\circ}$ & $-10^{\circ}$ & & $\mathrm{G}_{1 \mathrm{a}}$ & $82^{\circ}$ & $110^{\circ}$ \\
$\mathrm{C}_{\mathrm{a}}$ & $52^{\circ}$ & $80^{\circ}$ & & $\mathrm{G}_{2 \mathrm{a}}$ & $-158^{\circ}$ & $-130^{\circ}$ \\
\hline
\end{tabular}

Note.- Final point-on-wave or fault clearance angle correspond to voltages expressed with the cosine function. These angles must be increased by $90^{\circ}$ if the sine function is used. 
Table 1

Origin and transformation of voltage sags (from [11]-[12]).

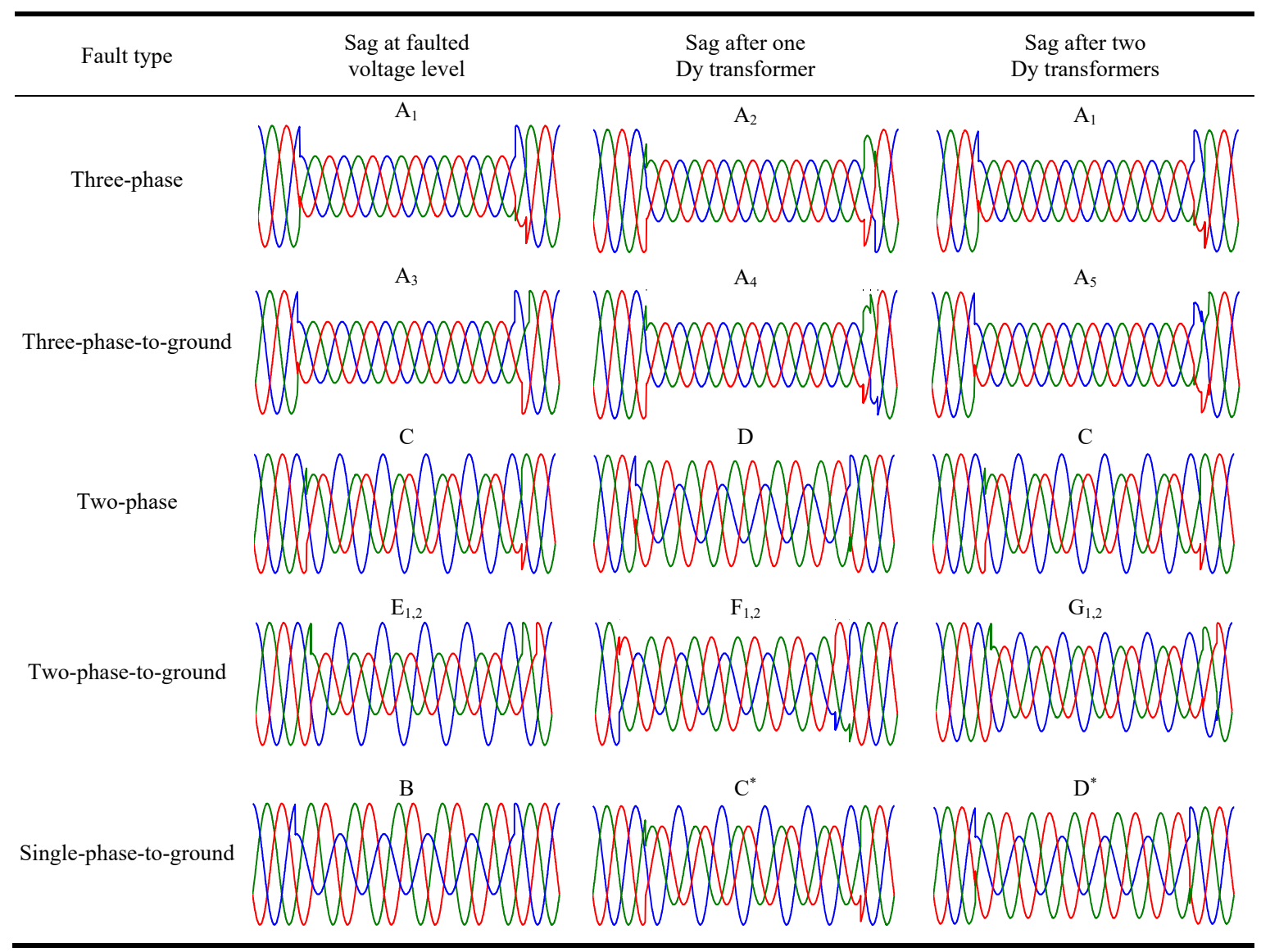

Table 3

Initial point-on-wave to obtain the most and the least severe effects at the instant of fault initiation. Sag depth $h=0.10$.

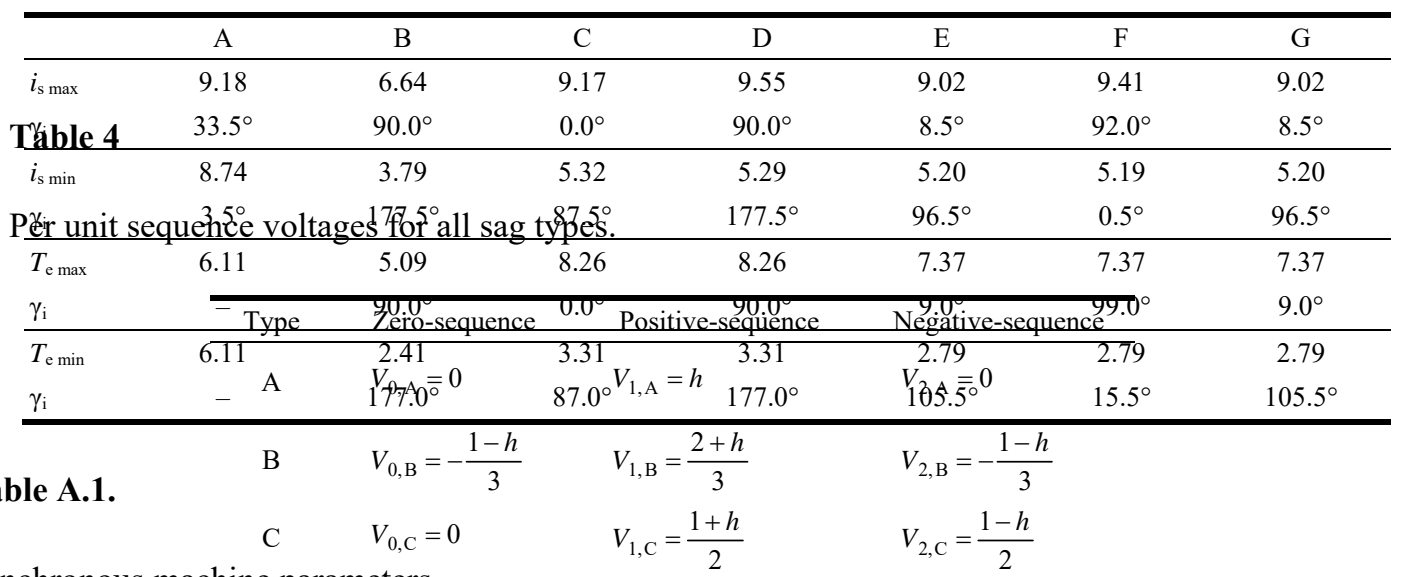

Synchronous machine parameters

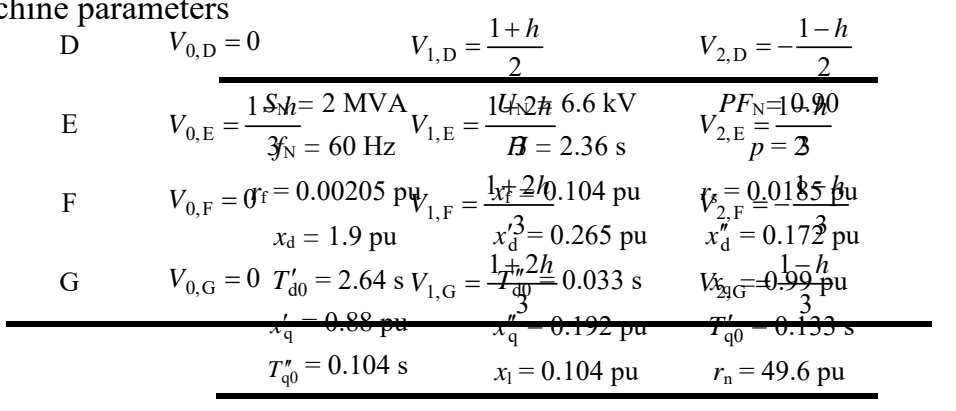



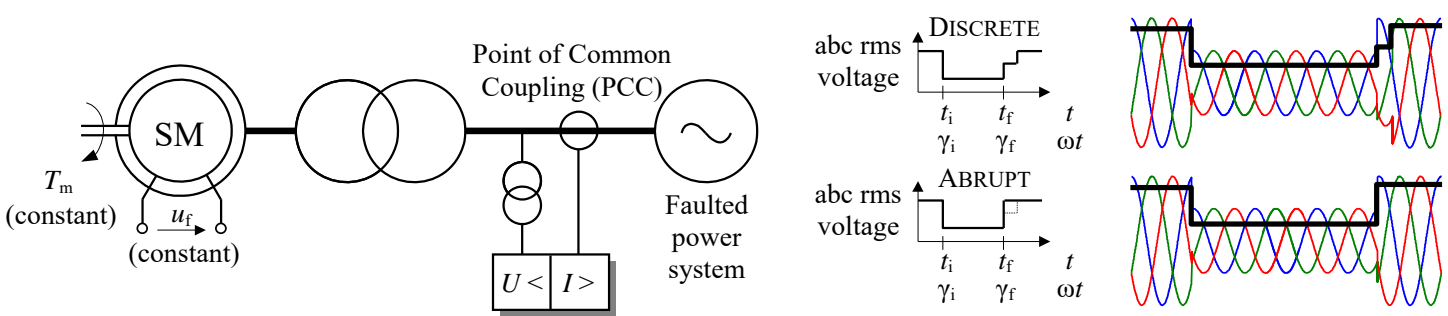

Fig. 1. Diagram of the studied system.
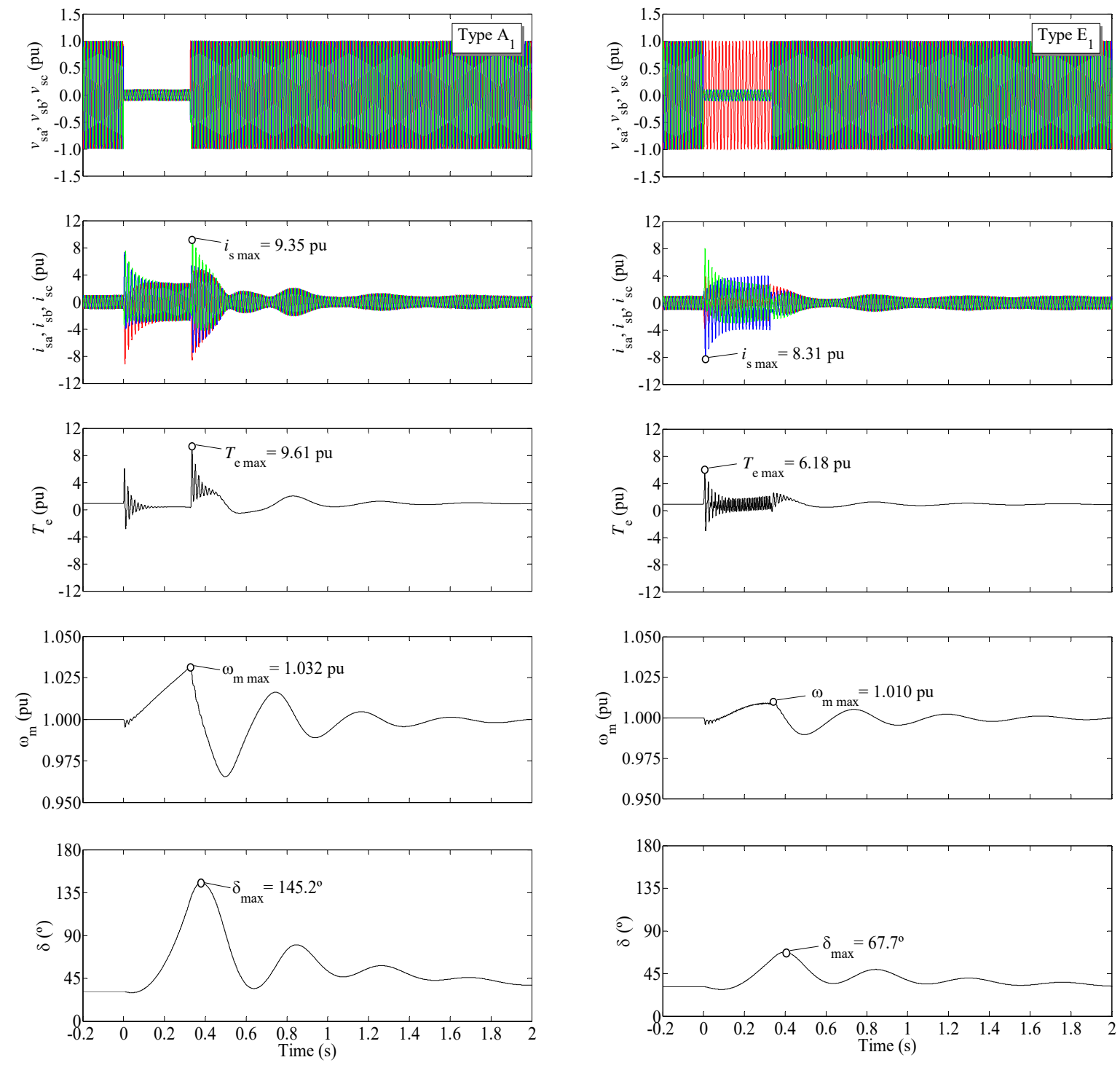

(a)

(b)

Fig. 2. Machine behaviour due to sags of characteristics. (a) Type $A_{1}, h=0.10, \Delta t=19.64 T$ (327.3 ms), $\psi=52^{\circ}$; (b) type $\mathrm{E}_{1}, h=0.10, \Delta t=19.81 T(330.2 \mathrm{~ms}), \psi=52^{\circ}$.

Symbols: $T_{\mathrm{e} \max }=$ maximum instantaneous torque; $\omega_{\mathrm{m} \max }=$ maximum instantaneous machine speed; $\delta_{\max }=$ maximum instantaneous load angle. 

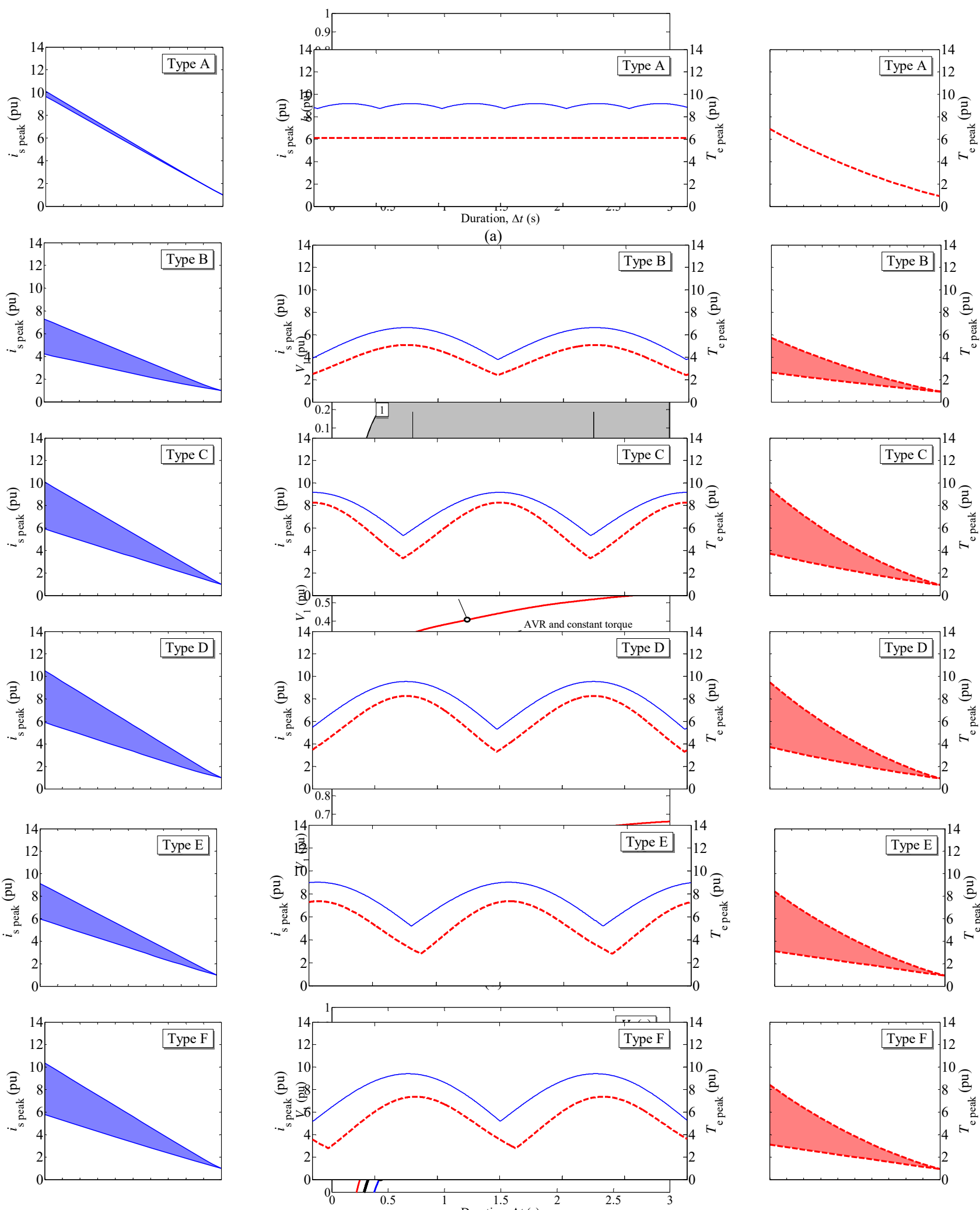

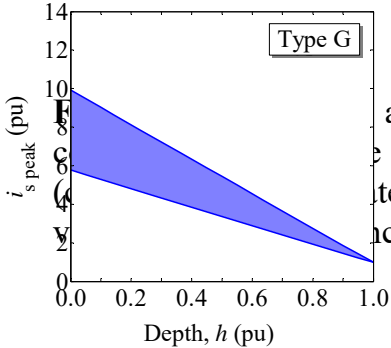

(a)

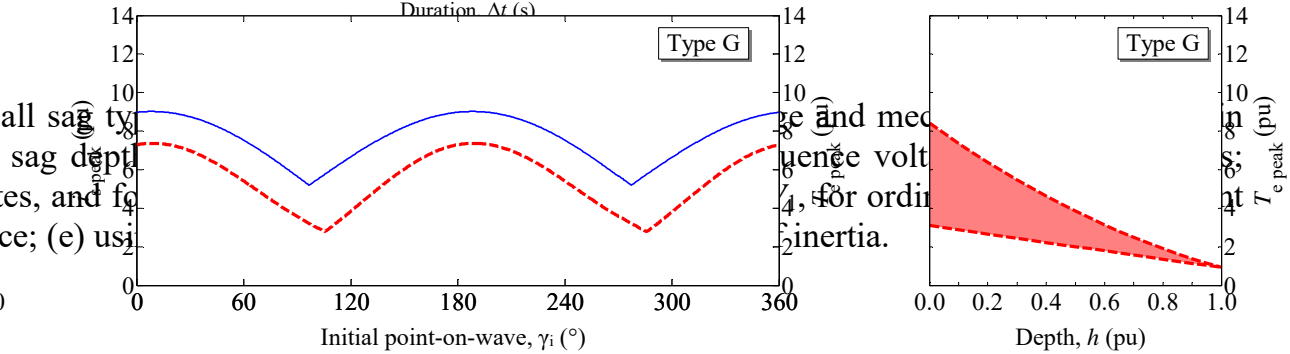

(b)

(c)

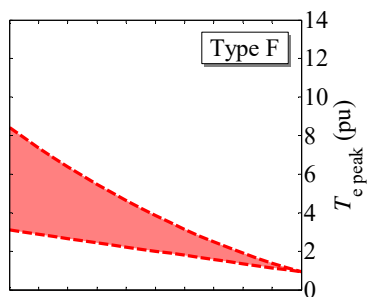

Fig. 3. Initial point-on-wave and depth influence on the current (solid blue line) and torque (dashed red line) peaks at sag initiation for all sag types. The initial point-on-wave influence is obtained for a depth $h=0.10$. 


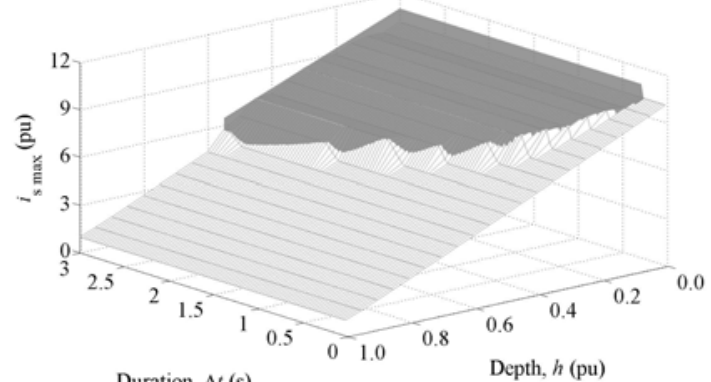

Duration, $\Delta t(\mathrm{~s})$

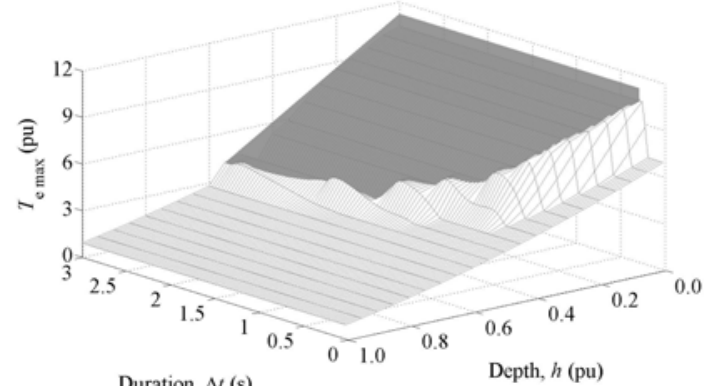

(a)
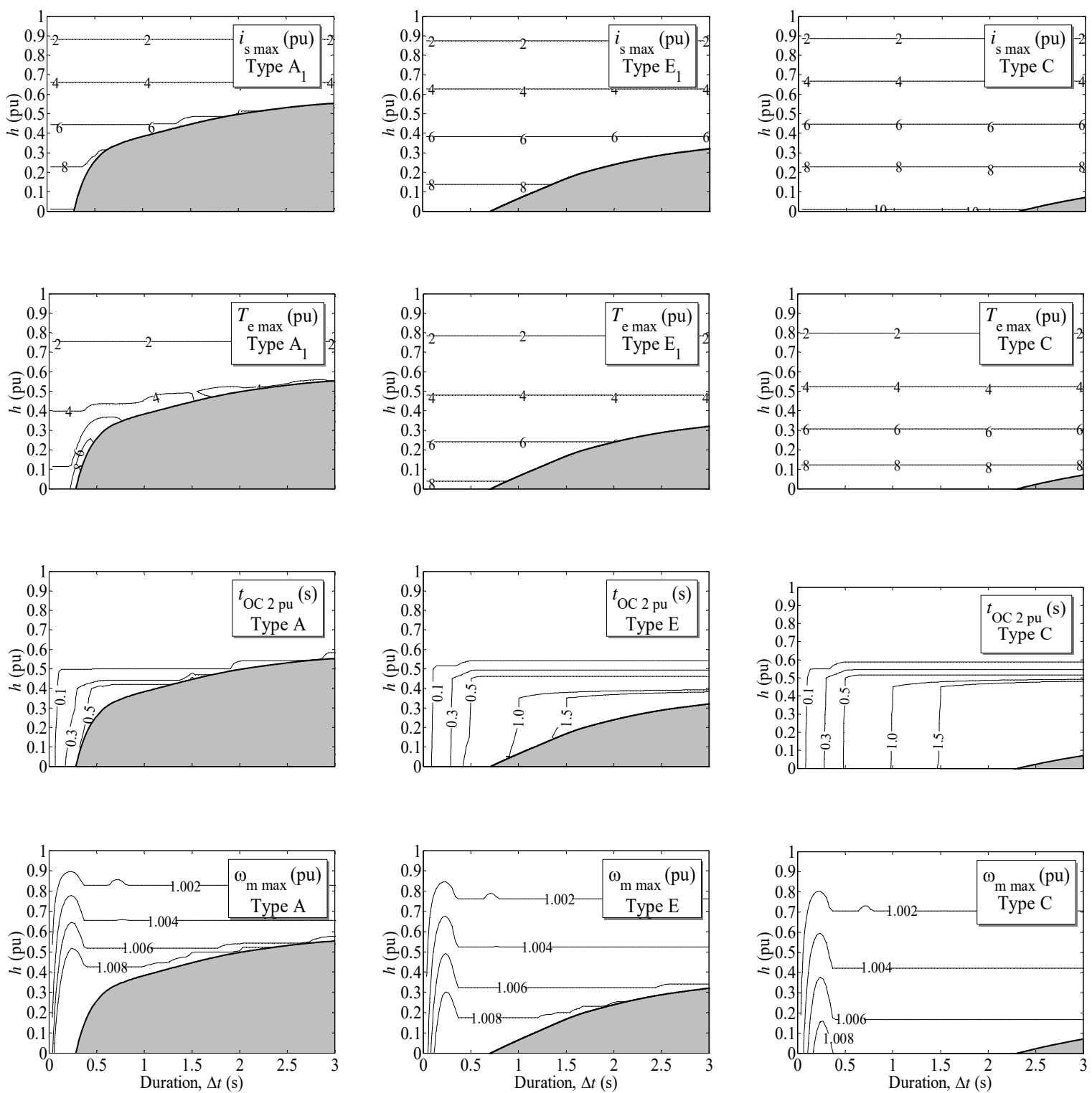

(b)

Fig. 5. Duration and depth influence. (a) Three-dimensional surfaces corresponding to current and torque peaks for sag type $A_{1}$, with $\psi=52^{\circ}$; (b) sensitivity curves for type $A_{1}$ symmetrical sags and for types $E_{1}$ and $C$ unsymmetrical sags, all with $\psi=52^{\circ}$. 


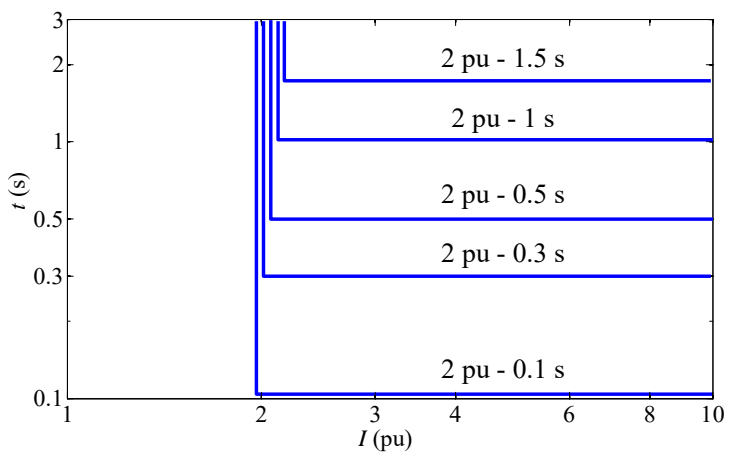

(a)

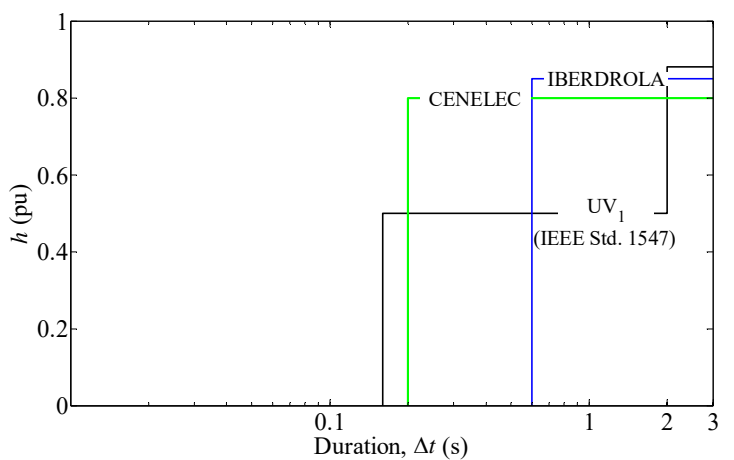

(b)

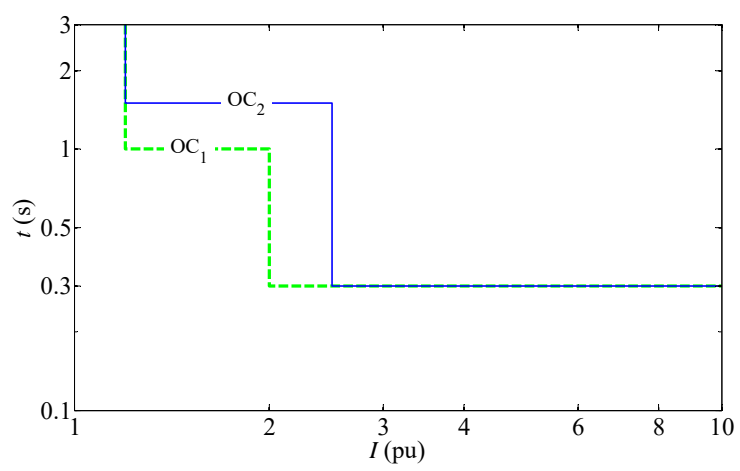

(c)

Fig. 6. Protection settings. (a) Settings for the definite time OC protection of Fig. 5; (b) undervoltage protection: current $\mathrm{UV}_{1}$ setting (IEEE Std. 1547) compared to Iberdrola and Cenelec settings; (c) overcurrent protection: current $\mathrm{OC}_{1}$ and proposed $\mathrm{OC}_{2}$ settings. 

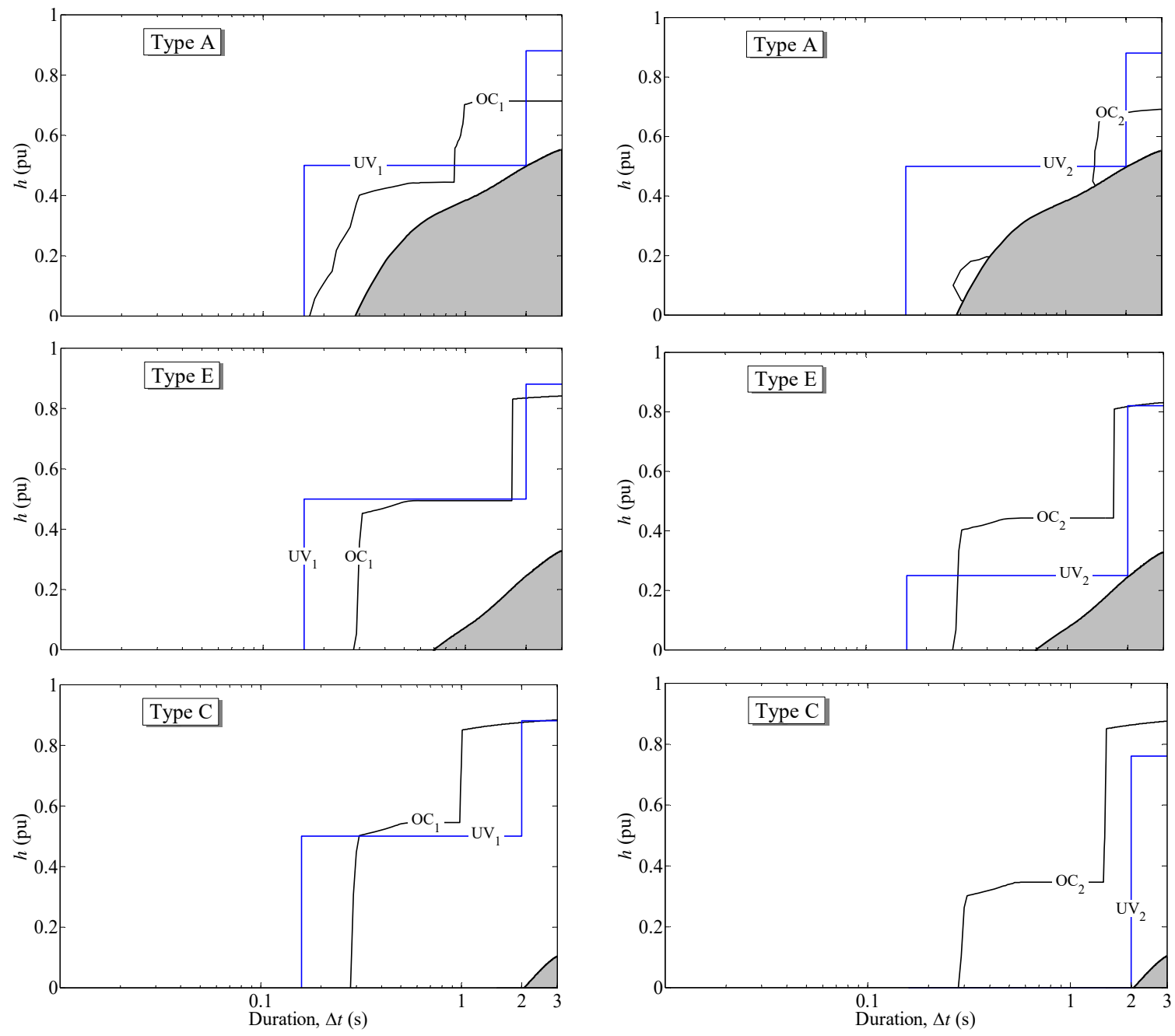

(a)

(b)

Fig. 7. Trip curves considering sag types $A, E$ and C. (a) Trip curves for current $\mathrm{UV}_{1}$ and $\mathrm{OC}_{1}$ settings compared to the CCT curves; (b) trip curves for the proposed $\mathrm{UV}_{2}$ and $\mathrm{OC}_{2}$ settings compared to the CCT curves.

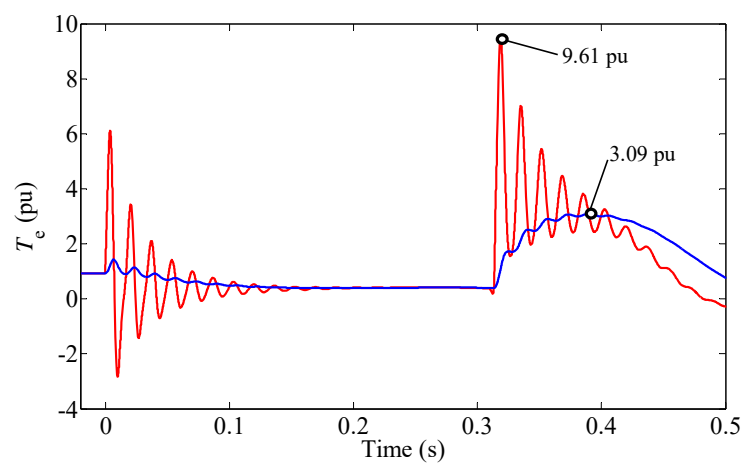

Fig. A.1. Detail of the instantaneous torque given by (A.1) and average torque calculated by PSCAD/EMTDC for the type $A_{1}$ sag in Fig. 2a. 